\title{
Amplifier circuit with LM324 operational amplifier Analytical calculation, Octave and Orcad LITE simulation
}

\author{
D.C.Puchianu
}

Valahia University of Targoviste, Faculty of Electrical Engineering, Electronics and Information Technology

E-mail: pdantgv@yahoo.com

Abstract. The study determines the time and frequency behavior of the LM324 operational amplifier. It determines the voltage amplification, the amplification given in $\mathrm{dB}$, the waveforms made with the help of the Octave and Orcad Lite simulator and the observation a fairly good correlation between the analytical calculation and the simulation media used.

Keywords: differential voltage, waveforms, cutoff frequency

\section{INTRODUCTION}

Operational amplifiers - are electronic direct current amplifiers. They are a category of analog amplifier integrated circuits with very good performance. Many linear and nonlinear applications can be realized [3]. The first types of operational amplifiers were used to perform certain simple mathematical operations (addition, subtraction, multiplication and division by a constant), hence the name "operational". [5]. Ideally, they can be characterized by infinite gain in voltage, infinite bandwidth, infinite input impedance and zero output impedance, but impossible to achieve. The actual amplifier is limited in terms of current and voltage, due to the realization techniques [1]. Thus the real amplifier is characterized by very high gain in voltage, very high input impedance, very low output impedance and a wide bandwidth.

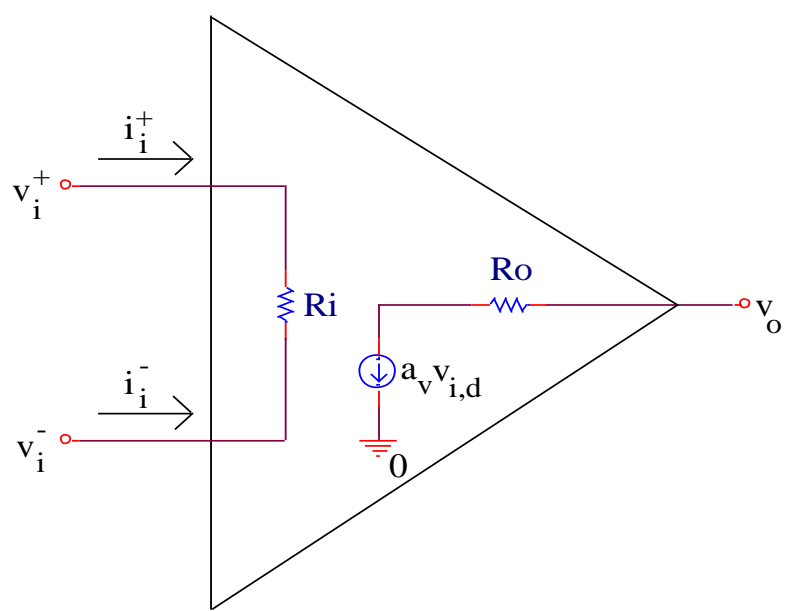

Figure 1. Operational amplifier - the general model.

The general model of the topology is shown in Figure 1, where $R_{i}$ is the input resistance of the infinite value amplifier, $R_{0}$ is the output resistance of value 0 and $a_{v}$ is the voltage amplification of infinite value. The values of the voltages on the input terminals are equal $v_{i}^{+}=v_{i}^{-}$, consequence of the negative reaction in the circuits with operational amplifiers, at which part of the output voltage is applied directly or indirectly on the inverting input by auxiliary elements. The operational amplifier is an active circuit that continuously modifies its output voltage until $v_{i}^{+}=v_{i}^{-}$, regardless of the configuration in which it is found in the circuit. [2] [4].

\section{AMPLIFICATION CIRCUIT WITH LM324}

In the case of the particular model, the scheme with operational amplifiers from Figure 2 is considered.

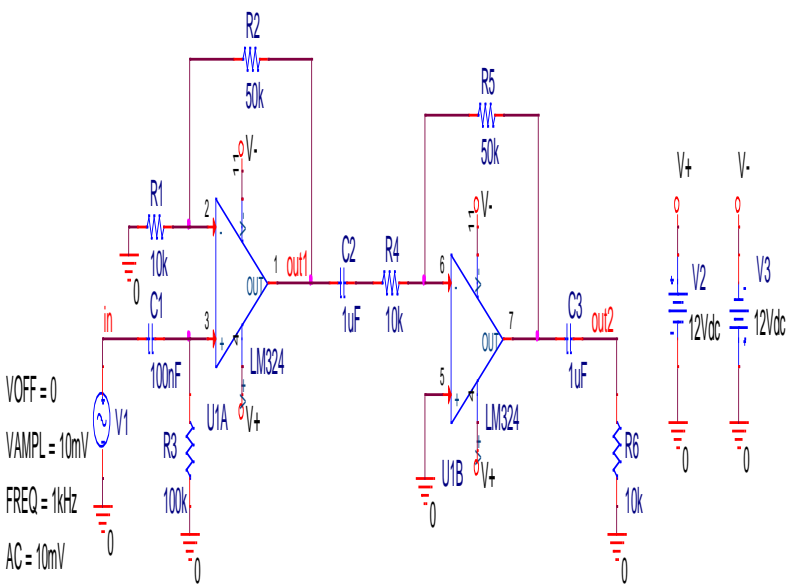

Figure 2. Amplification circuit with LM324.

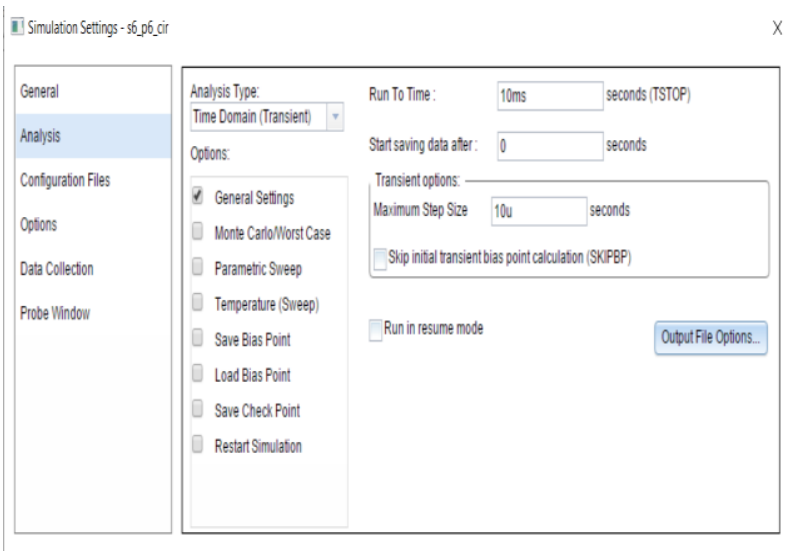

OK Cancel Appoly Resel Help

Figure 3. Amplifier time simulation parameters 
The behavior in time is considered for a time analysis over a period of $10 \mathrm{~ms}$, with a maximum step size of $10 \mu \mathrm{s}$, Figure 3.

The signal obtained at the output of the amplifier can be seen in Figure 4.

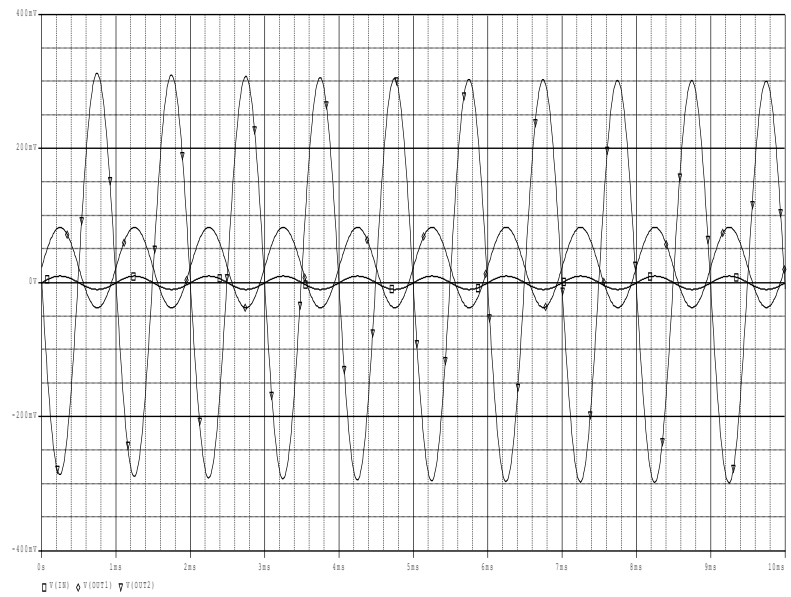

Figure 4. Amplification circuit with LM324 - time behavior.

2.1 Analytical calculation of amplifier output voltage $\left(v_{\text {out } 2}\right)$

The U1A operational amplifier is in non-reversing circuit configuration.

$$
\begin{gathered}
\left.\begin{array}{c}
A_{v 1}=1+\frac{R_{2}}{R_{1}} \\
A_{v 1}=\frac{v_{\text {out } 1}}{V_{1}}
\end{array}\right\} \Rightarrow \frac{v_{\text {out } 1}}{V_{1}}=1+\frac{R_{2}}{R_{1}} \Rightarrow v_{\text {out } 1}= \\
\left(1+\frac{R_{2}}{R_{1}}\right) \cdot V_{1}
\end{gathered}
$$

The U1B operational amplifier is in inverter circuit configuration.

$$
\left.\begin{array}{c}
A_{v 2}=-\frac{R_{5}}{R_{4}} \\
A_{v 2}=\frac{v_{\text {out } 2}}{v_{\text {out } 1}}
\end{array}\right\} \Rightarrow \frac{v_{\text {out } 2}}{v_{\text {out } 1}}=-\frac{R_{5}}{R_{4}} \Rightarrow v_{\text {out } 2}=-\frac{R_{5}}{R_{4}} .
$$

Then:

$$
\begin{gathered}
v_{\text {out } 2}=-\frac{R_{5}}{R_{4}} \cdot\left(1+\frac{R_{2}}{R_{1}}\right) \cdot V_{1} \\
v_{\text {out } 2}=-\frac{50 k \Omega}{10 k \Omega} \cdot\left(1+\frac{50 k \Omega}{10 k \Omega}\right) \cdot 10 \mathrm{mV} \cdot \sin \omega t \\
v_{\text {out } 2}=-5 \cdot 6 \cdot 10 \mathrm{mV} \cdot \sin \omega t \\
v_{\text {out } 2}=-300 \cdot \sin \omega t[\mathrm{mV}] \\
v_{\text {out } 2}=-300 \cdot \sin (2 \cdot \pi \cdot f \cdot t)[\mathrm{mV}]
\end{gathered}
$$

$$
T=\frac{1}{f}=\frac{1}{1 \mathrm{kHz}}=\frac{1}{1 \cdot 10^{3} \cdot \mathrm{Hz}}=10^{-3} \frac{1}{\mathrm{~Hz}}=10^{-3} \mathrm{~s}=1 \mathrm{~ms}
$$

$$
\frac{1}{H z}=S
$$

2.2 Octave simulation of the amplifier output voltage (vout 2$)$

$$
\begin{gathered}
f=1 k H z \Rightarrow v_{\text {out } 2}=\underset{(10)}{-300 \cdot \sin (2 \cdot \pi \cdot t)[m V]} \\
T=1 \mathrm{~ms}
\end{gathered}
$$

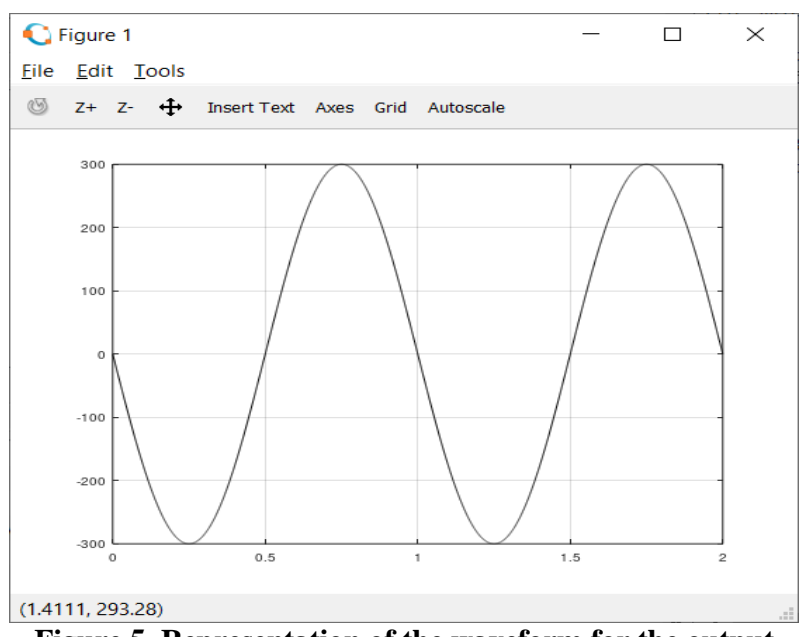

Figure 5. Representation of the waveform for the output voltage $v_{\text {out } 2}$ with Orcade.

2.3 Representation of the waveform of the amplifier output voltage $\left(v_{\text {out } 2}\right)$

$$
v_{\text {out } 2}(t)=-300 \cdot \sin (2 \cdot \pi \cdot t)[\mathrm{mV}]
$$

$t=0 \Rightarrow v_{\text {out } 2}(0)=-300 \cdot \sin (2 \cdot \pi \cdot 0)=-300$.

$\underbrace{\sin 0}_{=0}=0$

$t=0,5 \Rightarrow v_{\text {out } 2}(0,5)=-300 \cdot \sin (2 \cdot \pi \cdot 0,5)=$

$-300 \cdot \underbrace{\sin \pi}_{=0}=0$

$t=1 \Rightarrow v_{\text {out } 2}(1)=-300 \cdot \sin (2 \cdot \pi \cdot 1)=-300 \cdot$

$\underbrace{\sin 2 \pi}_{=0}=0$

$t=0,25 \Rightarrow v_{\text {out } 2}(0,25)=-300 \cdot \sin (2 \cdot \pi \cdot 0,25)=$
$-300 \cdot \sin (0,5 \cdot \pi)=-300 \cdot \underbrace{\sin \frac{\pi}{2}}_{=1}=-300$

$t=0,75 \Rightarrow v_{\text {out } 2}(0,75)=-300 \cdot \sin (2 \cdot \pi \cdot 0,75)=$

$-300 \cdot \sin (1,5 \cdot \pi)$

$v_{\text {out } 2}(0,75)=300 \cdot \underbrace{\sin \frac{3 \pi}{2}}_{=-1}=300$ 

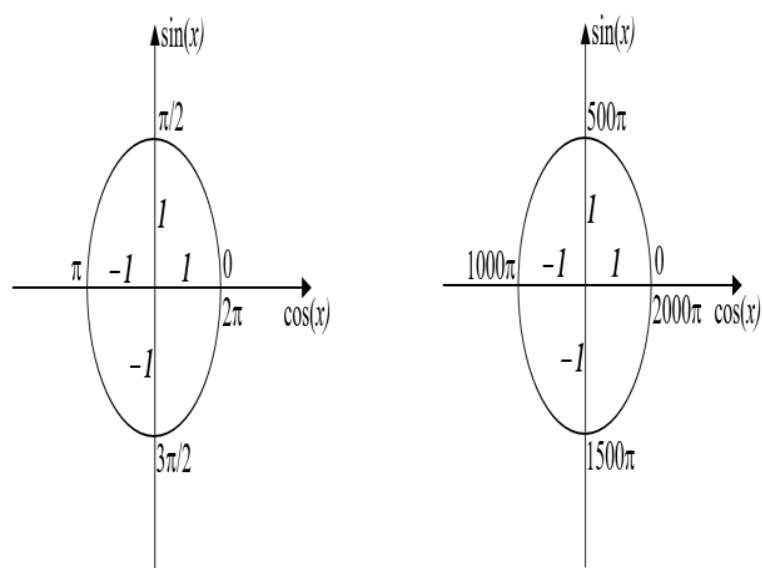

Figure 6. The values of the functions sin and cos on the trigonometric circle.

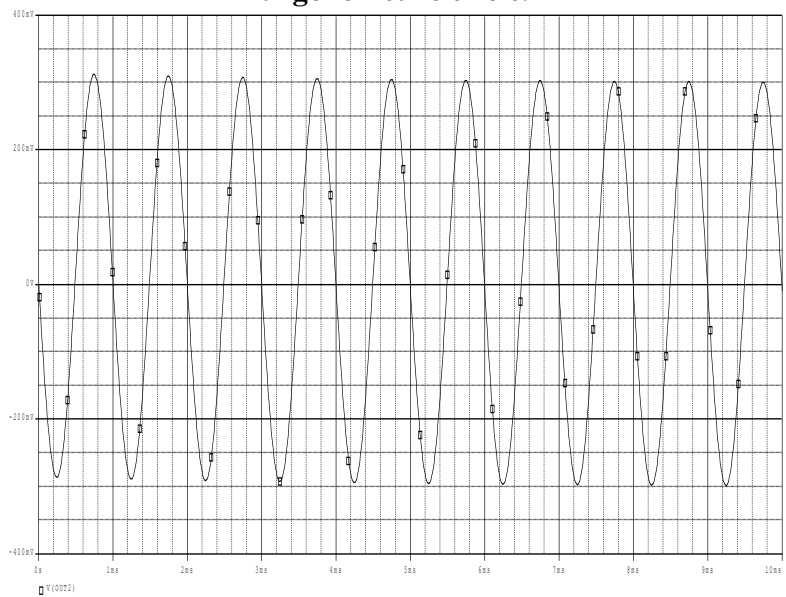

Figure 7. Waveform for the output voltage $v_{\text {out } 2}$.

\subsection{Determination of voltage amplification}

$\left(1+\frac{R_{2}}{R_{1}}\right)$

$$
A_{v}=\frac{v_{\text {out } 2}}{V_{1}}=\frac{v_{\text {out } 2}}{v_{\text {out } 1}} \cdot \frac{v_{\text {out } 1}}{V_{1}}=A_{v 2} \cdot A_{v 1}=-\frac{R_{5}}{R_{4}} .
$$

$$
A_{v}=-\frac{50 k \Omega}{10 k \Omega} \cdot\left(1+\frac{50 k \Omega}{10 k \Omega}\right)=-30
$$

$A_{d B}=20 \cdot \lg \left|A_{v}\right|=20 \cdot \lg |-30|=20 \cdot \lg 30=20$. $1,477=29,54 d B$

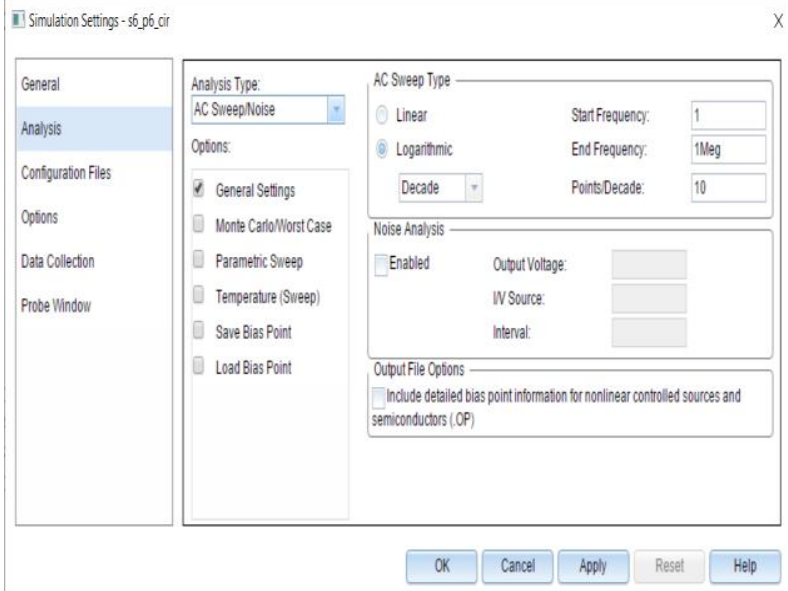

Figure 8. Frequency simulation parameters of the amplifier.

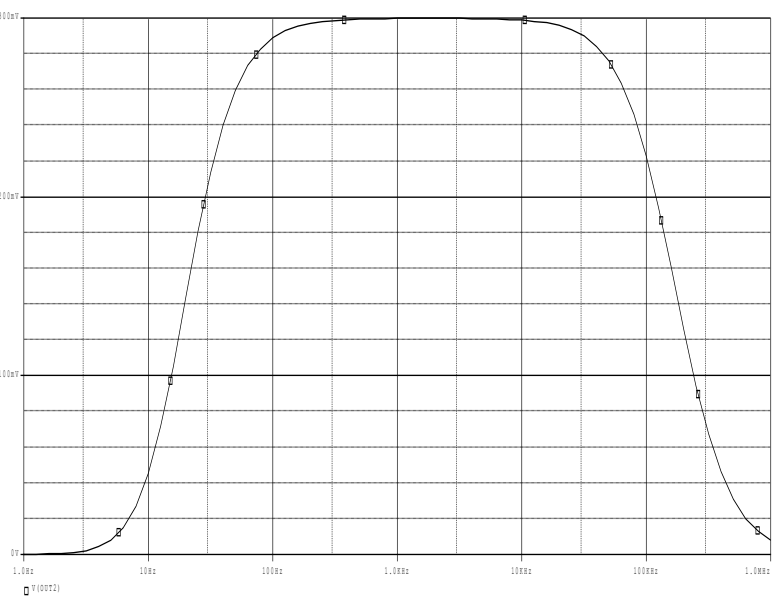

Figure 9. Frequency response of the amplifier.

The frequency response is given by the AC Sweep/Noise analysis for a start frequency of $1 \mathrm{~Hz}$, a stop frequency of $1 \mathrm{MHz}$ and 10 points per decade, Figure 8 and the frequency response of the amplifier is shown in Figure 9.

\section{Probe Cursor}

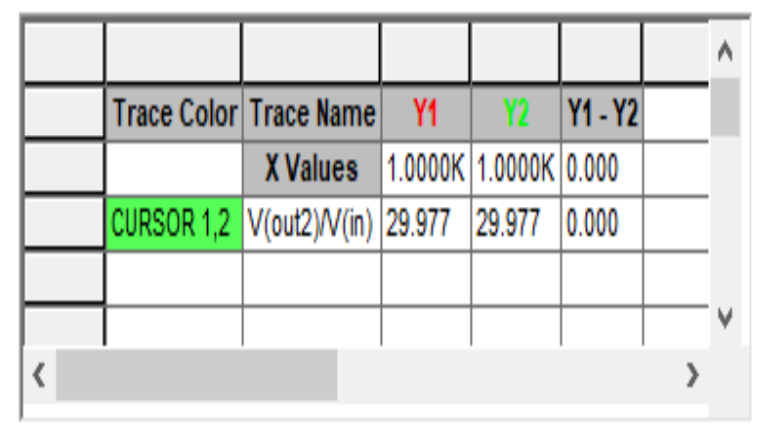

Figure 10. Spice amplification measurement.

2.5 Determination of the lower cutting frequency

$$
f_{T(\text { inf })}=\max \left(f_{T 1}, f_{T 2}, f_{T 3}\right)
$$




$$
\begin{aligned}
& f_{T 1}=\frac{1}{2 \cdot \pi \cdot R_{1} \cdot C_{1}}=\frac{1}{2 \cdot 3,1415 \cdot 100 \cdot 10^{3} \Omega \cdot 100 \cdot 10^{-9} F}= \\
& \frac{1}{2 \cdot 3,1415 \cdot 100 \cdot 100 \cdot 10^{-6} \Omega \cdot F} \quad(23) \\
& \quad f_{T 1}=\frac{1}{62830 \cdot 10^{-6}} \cdot \frac{1}{\Omega \cdot F}=\frac{10^{6}}{62830}[\mathrm{~Hz}]=15,91[\mathrm{~Hz}]
\end{aligned}
$$

$$
\begin{gathered}
F=\frac{1}{\Omega \cdot H z} \Rightarrow \frac{1}{\Omega \cdot F}=H z \\
f_{T 2}=\frac{1}{\frac{1}{2 \cdot \pi \cdot R_{4} \cdot C_{2}}=} \frac{1}{2 \cdot 3,1415 \cdot 10 \cdot 10^{3} \Omega \cdot 1 \cdot 10^{-6} F}= \\
\frac{(26)}{2 \cdot 3,1415 \cdot 10 \cdot 1 \cdot 10^{-3} \Omega \cdot F} \\
f_{T 3}=\frac{1}{62,83 \cdot 10^{-3}} \cdot \frac{1}{\Omega \cdot F}=\frac{10^{3}}{62,83}[\mathrm{~Hz}]= \\
15,91[\mathrm{~Hz}] \quad(27)
\end{gathered}
$$

\section{CONCLUSIONS}

There are many applications in which weak signals from different sources with specific impedances (often very high) must be amplified in the presence of external noises and high common voltages. In industrial, medical and automotive applications it uses sensitive circuits, which must remain immune to noise. Noise reduction can be done quite easily by using operational amplifiers.

\section{REFERENCES}

[1] Eugen Stefan Lakatos, Dan Constantin Puchianu, Modelare și simulare în SPICE. Manual de laborator, Valahia University Press, Târgoviște, ISBN 978606-603-140-0, 2015.

[2] Eugen Stefan Lakatos, Dan Constantin Puchianu, Proiectare în microelectronică, Valahia University Press, Târgoviște, ISBN 978-606-603-179-0, 2017.

[3] E. Diaconu, "Controlul sistemelor electronice", Targoviste, Romania, Valahia University Press, ISBN 978-606-603-170-7.

[4] Nicoleta Angelescu, M. Ionita, Dan Popescu, Alexandra Giuran, Dan Puchianu Application for detection and identifications of electronic components in preprocessed images; Scientific Bulletin of Electrical Engineering Faculty 2014 No. 2 ISSN 1843-6188, pag.11-14.

[5] Ion Vasile, Vicentiu Vasile, Emil Diaconu, Horia Andrei, Nicoleta Angelescu, Vital parameters monitoring system and alert signal transmission to Emergency Medical Centers" Journal of Science and Arts, no.3, 2019.
[4] Dan Constantin Puchianu, Ion Caciula, Horia Andrei, Emil Diaconu, Paul Cristian Andrei, „New approaches of nonlinear circuit elements analysis in frequency domain", Electronics, Computers and Artificial Intelligence International Conference ECAI 2017 -9th Edition, 29 June - 01 July, 2017, Târgoviste, ROMÂNIA, ISBN 978-1-5090-6458-8.

[5] Horia Andrei, Lucian Nastase, Emil Diaconu, Costin Cepisca, Sorin Dan Grigorescu, Paul Cristian Andrei, Contributions on Sensitivity Analysis for the Analog Two-Port Networks in Non-sinusoidal Regime, IEEE-EUROCON 2011 International Conference on Computer as a Tool - ConfTele 2011,27-29 April, 2011, Lisbon, Portugal, IEEE Catalog Number: CFP11EUR-CDR, ISBN 978-1-4244-7485-1, indexat IEEExplore/ISI Proceedings. 